\title{
HISTORIA
}

\section{LA ENSEÑANZA DE LA ENFERMERÍA EN LA ESPAÑA DEL SIGLO XVII. EL MANUAL DE ENFERMERÍA DE SIMÓN LÓPEZ (1668)}

Manuel Jesús García Martínez

Lic. en Antropología. D.U.E., H.U. Virgen del Rocío (Sevilla)

Antonio Claret García Martínez

Lic. en Geografía e Historia (Universidad de Huelva)
Figura 1

Portada del manuscrito Directorio de Enfermeros. La obra se divide en 8 Tratados y 150 capítulos con 587 páginas en total.
I

I DIRECTORIO. a?

DEENFER MEROS. YARTIFIÇE

Deobras de Caridad, para curarlas enfermeda des del cuerpo.

Conla pratica, desaner aplicar las Medicinas $\underline{\text {. }}$ ordenar los Hedicos, conelme Jor arte, y Thetfodo que aié ella.

Sequen los Doctores. Ana to mistas, que enseñany

Señalan, Las partes denues tro curze. donde seban de fiacer.

DíS PVESTO.

En ocbo Tratados, por simonloper, Barbero,de vn fospital íncognito.

DEDİ CADO.

Atodos los que có cari dad, desean Gacez esteo

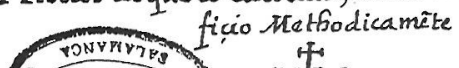

\section{THE TEACHING OR NURSING IN SPAIN DURING \\ THE $17^{\text {TH }}$ CENTURY. SIMON LOPEZ'S MANUAL (1668)}

\section{ABSTRACT}

The aim of the present study is to clearly state what nursing functions and tasks were in force in Spain in the 17th c. with emphasis on the teaching of nursing. The method used here has been based on the historical analysis of Simon Lopez's Manual: «Guidelines for nurses and volunteers for the caring of body illnesses» (1668).

The following conclusions have been reached: Simon López's work shows the existence of the four nursing functions which are recognized in nursing nowdays: care, teaching, administration and research. Lopez's work accurately describes the teaching activities carried out during that period. His text has been considered a key piece in the understanding of Spanish nursing in the 17 th $\mathrm{c}$. 


\section{INTRODUCCIÓN}

Al hablar de la historia de la Enfermería española, se suele aceptar por la mayoría de los investigadores como fecha clave de su institucionalización y profesionalización la de mediados del siglo pasado, y más concretamente la del año 1857, pues en el mismo se crean los títulos de Practicante y Matrona'. A partir de entonces irán surgiendo los correspondientes reglamentos y normas legales que regulan todo lo relativo a su enseñanza y campo de actuación. La información existente sobre la profesión enfermera desde dicho período es, en consecuencia, amplia, y los estudios llevados a cabo -aspectos legales, planes de estudios, textos empleados por enfermeras, practicantes y matronas... -numerosos y muy completos.

A medida que nos remontamos en el tiempo, las complicaciones son mayores, debido, fundamentalmente, a la escasez de documentación generada y conservada hasta nuestros días. De ahí la importancia de textos como el que nos ocupa, Directorio de Enfermeros (fig. 1), un manual para la instrucción de enfermeros, escrito por Simón López en el siglo XVII ${ }^{2}$. En dicha obra se encuentran ya reflejadas las cuatro funciones que en la actualidad se le reconoce a la profesión enfermera: asistencia, docencia, administración e investigación, siendo las dos primeras, con gran diferencia, las más ampliamente desarrolladas en aquélla.
El presente trabajo se plantea como objetivo analizar una de esas funciones de Enfermería, la docente, en ese período concreto, el siglo XVII ${ }^{3}$. La preocupación de Simón López por formar unos enfermeros responsables y bien instruidos constituye, como el propio autor reconoce repetidas veces a lo largo de la obra, el motivo principal por el que se tomó el trabajo de escribirla. Por último, deseamos llamar la atención sobre la importancia que adquiere en este tipo de estudio la consideración del contexto, de su ubicación temporal -siglo XVII- y espacial -en un hospital castellano-, en definitiva, de la realidad social en que se halla inmersa la obra, teniéndose en cuenta aspectos como la situación sanitaria, los conocimientos científicos existentes en esa época, reglamentación profesional y otros factores importantes.

\section{MATERIAL Y MÉTODO. UNAS PALABRAS SOBRE LA OBRA}

Para llevar a cabo el presente trabajo, se ha utilizado la obra Directorio de Enfermeros, cuyo título completo es Directorio de Enfermeros y artifiçe de obras de caridad, para curar las enfermedades del cuerpo. Con la práctica de sauer aplicar las Mediçinas que ordenan los Médicos, con el mejor arte, y Méthodo que ai en ella. Según los Doctores Anatomistas, que enseñan y señalan, las partes de nuestro cuerpo, donde se han de haçer.

1. La primera reglamentación de estas dos profesiones data de 1861 -Real Orden de 21 de Noviembre, publicada en la Gaceta de Madrid ${ }^{\circ} 332$, de fecha de 28 de Noviembre-. Puede consultarse al respecto nuestro trabajo: «Primer Reglamento en España para la enseñanza de Practicantes y Matronas (1861)». En Qalat Chábir (Revista de Humanidades), n², Julio de 1994. Alcalá de Guadaira (Sevilla), pp. 123-131.

2. Deseamos destacar, asimismo, y con la misma finalidad docente, el manual realizado por la Mínima Congregación de los Hermanos Enfermeros Pobres, Instrucción de Enfermeros, para aplicar los remedios a todo género de enfermedades, y acudir a muchos accidentes que sobreuienen en ausencia de los Médicos. Este tratado conocería cuatro ediciones entre los siglos XVII y XVIII; la segunda, llevada a cabo en Madrid, en la Imprenta Real, año 1625, por el Hermano Andrés Fernández, ampliaría notablemente el contenido de la primera, sirviendo de base para las ediciones posteriores. En sus primeras páginas o Prinçipio deste Tratado, su autor refiere lo siguiente: «He hecho este Tratado, para que los enfermeros tengan una doctrina llana, y bien assentada como va ésta, vista y apurada por tantos Médicos, tan doctos y experimentados, para que los escrupulosos cessen con sus escrúpulos, y tengan obligaçión a seguirla, pues con esto se les quitan todos, por ir tan apurada, porque en hauer yo tenido tantos, si en de mí los tengo bien allanados...».

3. Dada la importancia de esta obra para el conocimiento de la Enfermería española de los siglos XVI y XVII, hemos llevado a cabo su edición recientemente, con el patrocinio del Consejo General de Enfermería. Nuestro trabajo incluye la transcripción íntegra del texto, estudio introductorio, glosario de términos y un amplio índice de materias -11 en total-, con algo más de 2.800 entradas. Las citas hechas en el presente estudio están referidas a esta edición. 
Sobre su autor, Simón López, disponemos de pocos datos. Por las referencias contenidas en la obra, parece ser castellano de origen y enfermero y barbero de profesión, como reconoce él mismo en varias ocasiones. Hombre de gran cultura, a juzgar por las constantes referencias a autores clásicos médicos, botánicos, filósofos...-, tanto en castellano como en latín ${ }^{4}$, desarrolló el trabajo de enfermero durante casi tres décadas en distintos hospitales de Castilla $^{5}$.

El manuscrito ${ }^{6}$ consta de 565 páginas de texto, además de otras 22 que incluyen el índice, llamado Suma de todos los tratados deste libro de enfermeros, las correspondientes aprobaciones -tres en total, debidas a los doctores D. Juan de Río Noriega, catedrático de Anatomía de la Universidad de Salamanca, D. Juan Lázaro Gutiérrez, catedrático de Medicina de la Real Universidad de Valladolid, y D. Geromo Pardo, catedrático igualmente de la Real Universidad de Valladolid- y el interesante prólogo en el que se especifica el trabajo del enfermero en esa época, sus tareas y funciones.

El contenido se halla estructurado en ocho tratados, con un total de 150 capítulos. La distribución de éstos y los títulos de los tratados son los siguientes:

Tratado $\mathbf{1}^{\circ}$ (capítulos 1 a 9): «De las casos contingentes, en el qual se incluien todos quantos géneros de unturas ay y se pueden haçer en todo género de enfermedades con el Méthodo que combiene, según el buen orden de la Mediçina». Fig. 2.

Tratado $2^{\circ}$ (capítulos 20 a 33): «De otros casos contingentes en muchas enfermedades menudas, con algunos remedios en los prinçipios de ellas».

Tratado $3^{\circ}$ (capítulos 34 a 40): «De cómo se han de dar los xaraues y asistir a las sangrías y hechar los clisteres y supositorios o calas».

\section{Delasvntuzas.}

\section{TRATADO.}

Primero, de los casos, òreme dios cótingentes; Digo contingentes, Todos aquellas reme

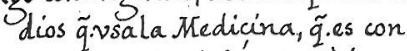
tingencía el hácerlos, ode xarlos de fracer.

Enelqual, seíncluien, todos quátos generos de vntuzas, sapue den facer, ento do genero, de é faimedades, con cl arte y.M.Methodo, $\bar{q}$. cóbune, segú reglas de buena me dicina.

\section{$\mathbb{C}_{0}$ Cap.1. $]$}

Delas zeglas a. seban deguardar, en facez to dogenerodernturas, 2 general mente, en.

todo genero de êfermedades.

Dasè primero, vna ad bertéciaj pasa hacezbiéntas vntuzas.

Para abxezuar, en oxden as eñalas, fition de múme bros, dódese ay an Lie hacer óaplicar vnturas, ostos xeme dior, lodire conla mayor bxiue dad, ybordad que pouduere, Siguion do onesto, al Dortistimo, Ju an devaluerde, de amusto, ensu Anatomiaidie,

\section{Figura 2}

El capítulo primero se dedica a la aplicación de las unturas. Este remedio terapéutico era de los más empleados dentro del trabajo enfermero.

Tratado $4^{\circ}$ (capítulos 41 a 56): «De las fluxiones de vientre con las disenterías propias y impropias».

Tratado $5^{\circ}$ (capítulos 57 a 108): «De las fiebres agudas y de las terçianas y quartanas, con el regimiento de ellas, y cómo nos emos de regir en tiempo de peste y preuençiones para ella». Fig. 3.

Tratado $6^{\circ}$ (capítulos 109 a 137): «De otras enfermedades, assí crónicas como ereditarias y acçidentales».

4. En el estudio que hemos llevado a cabo sobre Directorio de Enfermeros, se han contabilizado un total de 92 referencias a obras de distintas materias: medicina, cirugía, botánica... El número de autores citados ronda el medio centenar.

5. En la portada de Directorio de Enfermeros se afirma lo siguiente: «Dispuesto En ocho Tratados, por Simón López, Barbero, de un hospital incógnito».

6. GARCÍA MARTÍNEZ, M. J.; GARCÍA MARTÍNEZ, A. C.; VALLE RACERO, J. I. (1995): «Ficha Bibliográfica n.1 5: Directorio de Enfermeros». En Híades. Revista de Historia de la Enfermería, n 2, Septiembre de 1995. Alcalá de Guadaira (Sevilla), pp. 101-110. 


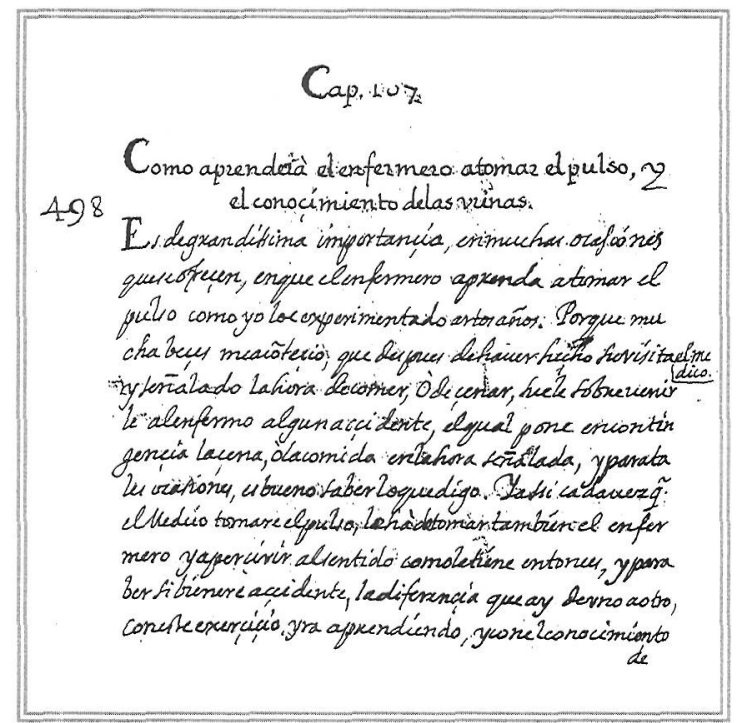

Figura 3

La técnica de la toma del pulso se explica en el capítulo 107. En el mismo se advierte que su conocimiento era «de grandísima importancia»

Tratado $7^{\circ}$ (capítulos 138 a 148): «De la calidad del agua y quál es la mexor, y de todo género de aguas coçidas que beuen los enfermos y el modo de coçerlas con su peso y medida».

Tratado $8^{\circ}$ (capítulos 149 y 150): «De la calidad de los mantenimientos simples que nos sirben para nuestra conseruaçión».

El trabajo del enfermero de la época queda expuesto a lo largo de toda la obra, pero muy especialmente en su Prólogo. Muy resumidamente tenemos las siguientes funciones y tareas:

$\checkmark$ Ejecutar las prescripciones ordenadas por el médico.

Observar al enfermo; ver su evolución e informar de la misma al médico.

$\checkmark$ Proveer todo lo necesario para las curas.

Higiene y limpieza de las enfermerías y aposentos, con especial atención a su ventilación.

Asistencia al enfermo en situaciones de urgencia en ausencia del médico o cirujano.
Aplicar unturas; administrar ayudas o clisteres; dar jarabes y bebidas cordiales; dar purgas; hacer embrocaciones, fregaciones y ligaduras; dar garrotes.

$\checkmark$ Administrar las comidas, con la debida higiene.

Otras varias tareas.

El primer borrador de la obra está fechado en 1651 y el definitivo, que recoge las sugerencias y correcciones oportunas, en 1668.

\section{DIRECTORIO DE ENFERMEROS Y LA ENSEÑANZA DE LA ENFERMERÍA}

La función docente, entendida como la capacidad de acción orientada a instruir y educar, con el fin de dotar a los enfermeros de los conocimientos teóricos y prácticos necesarios para el correcto ejercicio de la profesión ${ }^{7}$, se halla plenamente desarrollada a lo largo del manual.

La obra Directorio de Enfermeros ha sido concebida, fundamentalmente, para la formación del enfermero, tanto del que se iniciaba - «prinçipiante»- como de aquel que ya se encontraba ejerciendo el ofiçio. En dicha formación se incluían el conocimiento de aquellas técnicas, tareas y actividades propias de la profesión -embrocación, ligaduras...-, y la preparación del enfermero para situaciones de emergencia en ausencia del médico -hemorragias, caídas con pérdida del conocimiento y otros accidentes-.

La insigne profesora Virginia A. Henderson definía la profesión enfermera, hace tres décadas, con las siguientes palabras: «Creo que la enfermera es, y debe ser legalmente, un profesional independiente capaz de hacer juicios independientes mientras no haga diagnósticos, prescriba tratamientos o emita pronósticos, porque éstas son las funciones del médico. Pero la enfermera es la mayor autoridad en los cuidados básicos de enfermería. Quizá deba explicar que por cuidados básicos de enfermería me refiero a ayudar a los pacientes en las siguientes actividades o darles los conocimientos necesarios pa-

7. HERNÁNDEZ MARTÍN, F. (Coordinadora) (1996): Historia de la Enfermería en España (desde la Antigüedad hasta nuestros días). Editorial Síntesis, S. A. Madrid, p. 205. 
ra que puedan llevarlas a cabo sin ayuda [se nombran 14 actividades]» ${ }^{8}$. Este interés por formar e informar al paciente en determinadas cuestiones relacionadas con su patología, con la finalidad de que éste se valiese por sí mismo lo antes posible, contribuyendo, por tanto, a la resolución de su enfermedad, se halla igualmente presente en el tratado que estudiamos, como tendremos ocasión de comprobar -recuérdese que éste se escribió tres siglos antes de la teoría de Henderson-. Por último, el interés docente de Simón López se hace extensible, también, a la comunidad donde se desarrolla el individuo; ello se manifiesta en forma de consejos sanitarios o el empleo de determinadas medidas ante situaciones de contagio y propagación de enfermedades infecciosas -peste, viruelas, sarampión....Como se ha referido anteriormente, el interés por formar al enfermero, porque éste adquiriese los conocimientos necesarios, tanto los relativos a su propia disciplina como sobre otras diversas materias (Medicina, Botánica, Dietética...) que se hacían imprescindibles para el ejercicio del oficio, constituye el objetivo fundamental planteado por Simón López. Ello queda patente ya desde las páginas prologales:

«... No mires quien lo escriuió, sino mira qué tal es lo escrito, que a mí el buen çelo que siempre tube de la salud y alibio de los enfermos y la larga experiençia de muchos años de enfermero me fue motiuo para tomar este trabaxo y, ansimismo, el ber y experimentar quán pocos son los que en estos tiempos procuran de preçiarse de buenos enfermeros, siendo de tanta importançia en la Mediçina i de tan grande seruiçio de Dios y mérito de los enfermeros, como adelante se prouará. Para lo qual, procuren, con la graçia de Dios y para instruir a los que comiençan a exerçer este ofiçio de enfermero, el buscar y inquirir el mejor méthodo y la mejor orden de sauer aplicar las Mediçinas...»".

Este texto refleja algunas de las ideas que Simón López irá exponiendo y ampliando a lo largo de las páginas de su Directorio, tales como su objetivo al escribirlo ( $\ll .$. para instruir a los que co- miençan a exerçer este ofiçio»), la diferencia entre buenos y malos enfermeros ( «... quán pocos son los que en estos tiempos procuran de preçiarse de buenos enfermeros») y la importancia de la Enfermería en el conjunto de las profesiones sanitarias.

Al tratarse de una obra con una finalidad eminentemente docente, su autor emplea un lenguaje claro, conciso, fácil de entender, con frecuentes empleos de recursos didácticos, como el ejemplo, la comparación y otros que, en definitiva, permitían al futuro enfermero una más fácil captación y comprensión de los conocimientos que se desean transmitir. Lo anteriormente manifestado adquiere más relieve al tratarse la Enfermería de una profesión fundamentalmente práctica, en donde es importante unas habilidades para llevar a cabo una determinada técnica.

Detengámonos unos instantes en este punto $\mathrm{y}$ observemos ese lenguaje preciso, detallado, que el enfermero Simón López emplea habitualmente:

«Para que la orina no pase los colchones, se ha de usar de unas mantas berriondas pardas, muy peludas, que las haçen en Palençia y en Valladolid, que suelen servir de arpilleras para traer las otras mantas buenas» ${ }^{10}$.

Como puede apreciarse, no sólo se le enseña al enfermero el material que debía emplear para evitar que la orina traspasase el colchón, sino que se especifica, exactamente, el tipo y lugar donde se fabrica -han de ser «mantas berriondas pardas», de las fabricadas en Palencia y Valladolid-. Como nota aclaratoria, advertimos que el término «mantas berriondas» -en nuestro tiempo, verriondas- alude a un determinado tipo de mantas más duras y ásperas que las normales, cuya función es la de servir de protección -de «arpilleras»-, precisamente, a las de mayor calidad o al colchón mismo.

El empleo de refranes, sentencias y dichos populares es, asimismo, frecuente en las páginas de Directorio de Enfermeros:

8. HENDERSON, V. A. (1994): La Naturaleza de la Enfermería. Reflexiones 25 años después. Interamericana-McGraw-Hill. Madrid, pp. 22-23.

9. GARCÍA MARTÍNEZ, A. C.; GARCÍA MARTÍNEZ, M. J.; VALLE RACERO, J. I. (1997): Directorio de Enfermeros. Simón López. Organización Colegial de Enfermería. Consejo General. Editorial Síntesis, S. A. Madrid, p. 86.

10. Ibídem, p. 195. 
«... sobre lo qual traen una sentençia los Médicos, diziendo: Fiebres otoñales, largas o mortales»".

«Y de aquí emanó el adagio bulgar que dize: Agua buena, sin color, olor, ni sabor y que la bea el sol»!2.

La obra no se halla exenta, en determinadas situaciones, de un lenguaje humorístico, que esconde un agudo sentido crítico del autor:

«En ensuçiándose todo esto, se le mudará otro tanto, y deste modo hasta que se acabe la fluxión o el enfermo [en caso de diarrea]» ${ }^{13}$.

$\ll \mathrm{E}$ bisto muchas beçes la ignorançia que en esto tienen algunos, que por raçón de su ofiçio debrían saberlo, que me pareçió abrirles los ojos (y aquí no con cáusticos) con raçones...» ${ }^{14}$.

Para que el contenido del mensaje se recibiese en su integridad y fuera comprendido por parte del aprendiz o enfermero, se recurre frecuentemente al uso de la comparación, utilizándose para ello objetos conocidos y de uso diario -la corneta de ministril o la moneda vigente en esa época-:

«Para hechar estos poluos por las narizes a estos enfermos, es menester usar de una caña de jazmín gueca, de largo de media bara y de la figura de una corneta de ministril [aplicación de un estornutatorio]» ${ }^{15}$.

«Para aplicar estos cáusticos de cantáridas, se ha de preuenir primero un poquito de baldrés o baldana, del tamaño y redondo de un real de a quatro segobiano.... ${ }^{16}$.

Otro recurso didáctico empleado repetidas veces es el ejemplo. Ello contribuía a facilitar la comprensión de los conocimientos teóricos y su posterior aplicación en la práctica:

«Pongamos un exemplo de un enfermo que tiene calentura con creçimientos y le biene el frío o creçimiento a las dos de la tarde, el qual puede benir tam- bién sin frío... [para calcular la hora en que el enfermero debía aplicar un determinado remedio en el caso de la fiebre]» ${ }^{17}$.

«Pues bamos a la prueua con un exemplo. Si esta ropa suçia la pusiesen a un sano, es çierto que enfermaría con su contacto... [necesidad de cambiar la ropa contaminada al enfermo]» ${ }^{18}$.

En su afán didáctico, en ocasiones, el autor iba más allá de la mera exposición teórica de una determinada técnica, aconsejando, en base a su larga experiencia acumulada como barbero y enfermero, la construcción de un objeto que sirviera para una finalidad concreta. La siguiente cita nos muestra el material a emplear y los pasos a seguir en la construcción de un instrumento para limpiar la suciedad de la lengua:

«Diré aquí una muestra para que por ella se saque cómo han de ser las baçiadas de plata o de latón. Tome una tirica de hoja de lata, de largo de siete dedos de trauessía y del ancho de un anillo de una tixera de barbero, y dóblela haçiendo de ella como una $\mathrm{D}$, y junte las dos puntas, las quales clauará con dos tachuelas en un palillo de un palmo, y que sea quadrado por donde se ha de clauar; después de clabado, le dará forma de una $\mathrm{D}$, como es dicho, y con este instrumento rallé yo artas beçes las lenguas a los enfermos algunos años...» ${ }^{19}$.

\section{Enseñanza de una técnica o procedimiento de Enfermería}

Analizando la metodología empleada en la obra a la hora de exponer una técnica concreta o la realización de una tarea, nos llama la atención la gran similitud que encontramos con la llevada a cabo en: la actualidad; nos referimos a los distintos pasos que debía seguir el enfermero, desde el inicial, es decir, informar al paciente sobre la técnica en cuestión, hasta la finalización de la misma, contem-

11. Ibídem, p. 259.

12. Ibídem, p. 327.

13. Ibídem, p. 195.

14. Ibídem, p. 132.

15. Ibídem, p. 218.

16. Ibídem, p. 131.

17. Ibídem, p. 262.

18. Ibídem, p. 148.

19. Ibídem, p. 216 . 
plándose, incluso, las posibles complicaciones o efectos secundarios que podrían derivarse de su aplicación:

- Es importante que el enfermero sepa lo que hace y por qué lo hace:

«Aora digamos las raçones que ay para haçer esto desta manera y no de otra ${ }^{20}$.

«... porque de qualquiera otra suerte que se haga, siempre se haçe llamamiento.... ${ }^{21}$.

- Adopción de las correspondientes medidas higiénicas -en el siguiente caso, el lavado de manos-:

«... y estése assí en el agua por espaçio de hora y media o dos, y al cabo de ellas (y labándose las manos primero), frotarán y extregarán con ellas la taleguilla [para preparar una talvina]” ${ }^{22}$.

- Preparación y puesta a punto del material antes de la ejecución de la técnica:

«De lo que se ha de preparar antes de hechar los clisteres.... ${ }^{23}$.

«Antes de dar la purga, ha de preuenir vinagre blanco o tinto..., y un lienço para mojarlo en él, y un palillo para rebolber la purga quando se la ba a dar, y un baso de agua para enjuagarse después de tomada la purga, y una almofía... ${ }^{24}$.

«Lo que se ha de cautelar antes de haçer los remedios... [en la gota coral] ${ }^{25}$.

- Los conocimientos anatómicos se consideraban imprescindibles para llevar a cabo cualquier trabajo enfermero:

«Y para sauer dónde está el hígado, dígale que ponga los dedos de la mano derecha ençima de la boca (assí como quando haçemos cortesía a otro) y, a donde cayere el codo, allí está situado el hígado. Y la mesma diligençia se ha de haçer con la mano hizquierda para sauer dónde está situado el baço» ${ }^{26}$.

La administración de la purga era una tarea habitual, cotidiana, en el conjunto del trabajo enfermero de ese siglo. De hecho, la purga, junto a la sangría, constituían los llamados remedios mayores de la Medicina ${ }^{27}$, siendo prescritos por los médicos en infinidad de procesos patólogicos y accidentes varios. Los capítulos 133, 134 y 135 de Directorio de Enfermeros se dedican a explicar la técnica de su administración, de forma clara y pormenorizada, de tal manera que su exposición puede servirnos como modelo de metodología empleada por Simón López. A continuación se muestran, de forma resumida, los pasos seguidos:

1.- Prescripción de la purga por parte del médico. La advertencia en el cumplimiento de este punto es una constante en toda la obra.

2.- Preparación del enfermo el día anterior (administración de una ayuda, cena ligera, reposo).

3.- Medidas a tomar por el enfermero el día en que se llevaba a cabo su aplicación (preparación del material, entrevista con el enfermo, dieta adecuada).

4.- Ejecución de la técnica (postura del enfermo, medidas preventivas para evitar enfriamiento, toma del preparado correspondiente, enjuague posterior con agua o vino, acostarle algo incorporado y cubierto de ropa si es invierno, prevención del vómito con la aplicación de un paño mojado en vinagre).

5.- Medidas a tomar tras la realización de la técnica (administrar al paciente media cucharada de

20. Ibídem, p. 132.

21. Ibídem, p. 130.

22. Ibídem, p. 243.

23. Ibídem, p. 171.

24. Ibídem, p. 317.

25. Ibídem, p. 313.

26. Ibídem, p. 95.

27. A este respecto, el historiador de la Medicina Anastasio Rojo Vega, al tratar la terapéutica en los siglos XVI y XVII, afirma lo siguiente: «El objetivo fundamental [de la Terapéutica] es ayudar al enfermo a eliminar la materia que le contamina -la lavativa que ayuda a defecar se llama precisamente ayuda- $\mathrm{y}$, por ello, la terapéutica médica más utilizada es la evacuante; sangría y purga son los remedios habituales. No hay enfermedad en que no se hagan sangrías -cuando hay médico por delante-, y las purgas faltan pocas veces...». ROJO VEGA, A. (1993): Enfermos y sanadores en la Castilla del siglo XVI. Secretariado de Publicaciones de la Universidad de Valladolid. Salamanca, p. 67. La obra de Simón López viene a confirmar plenamente lo afirmado en estas líneas. 
anís y tres aceitunas sevillanas, por ejemplo, todo ello con el objetivo de retener la purga; no dejarle dormir; masajear sucesivas veces la región epigástrica, con la finalidad de que defeque el enfermo lo antes posible).

6.- Medidas a tomar tendentes a prevenir los efectos secundarios (aplicar un clister, administrar un caldo...).

7.- Finalmente, se le enseña al enfermero los distintos tipos de purgas (çiuales, lenitiuas, minoratiuas...), así como su composición y forma de administración y aquellas enfermedades en que se suelen emplear (fiebres terçianas dobles, sençillas...).

Al margen de los resultados obtenidos, los cuales guardarán relación directa con los conocimientos científicos existentes en esos momentos -recordemos que se desconocían, por ejemplo, los efectos secundarios de muchos medicamentos o la etiología y mecanismos de transmisión de las enfermedades infecciosas-, llamamos la atención sobre el procedimiento metodológico llevado a cabo por el enfermero, justificándose cada paso seguido.

\section{ENSEÑAR AL ENFERMO. EL AUTOCUIDADO}

Se comentaba anteriormente, en relación a la definición de Enfermería de la autora Virginia A. Henderson, la necesidad de que el paciente colaborase en el proceso de su curación o rehabilitación lo antes posible. En este sentido, el papel del enfermero es central, ayudándole, bien transmitiéndole información, fundamentalmente sanitaria, sobre su patología, bien explicándole la realización de una técnica determinada. A continuación se muestran algunos de estos aspectos:

«Y si esto puede haçer el enfermo, le enseñará el enfermero cómo lo ha de haçer, teniendo cuidado de renouarle a menudo las dos escudillas o basos de agua fría.... ${ }^{28}$.
«... y enseñar al enfermo cómo ha de tomar los lamedores y cómo ha de açer los gargarismos y otras cosas que aora se dirán que le tocan, de todo lo qual ya dexo dicho arto en sus lugares donde podrá recurrir, por no me detener aquí más» ${ }^{29}$.

En el primer caso, se trata de que el enfermero enseñe al paciente cómo se construye un hisopillo de zaragatona y pepitas de membrillo para limpiar las asperezas de la lengua; dicha recomendación era especialmente útil en enfermos que presentaban algún tipo de trastorno psiquiátrico - «enfermos delirantes»-. La segunda cita hace referencia al papel educativo del enfermero cuando el paciente debía tomar un lamedor (composición que se hacía en la botica de varios simples con azúcar, de menor consistencia que el electuario y de más que el jarabe) o hacer gargarismo (indicado en la enfermedad del garrotillo o difteria laríngea).

$\mathrm{Al}$ igual que sucede hoy día con los programas de educación para la salud, en Directorio de Enfermeros se considera de gran importancia las prescripciones dietéticas, destacándose su papel tanto en la prevención como en la cura de las enfermedades:

«Es de grande importançia el gobierno en las comidas y demás cosas que se administran y hechas a saçón a todo género de enfermos...» ${ }^{30}$.

«... la buena regla en estos enfermos es mediçina preseruatiua, guardándose de comidas agudas y saladas y mucho vino [consejos para el enfermo de gota y ciática]» ${ }^{31}$.

\section{EDUCACIÓN SANITARIA Y COMUNIDAD. EL PAPEL DE ENFERMERÍA}

Por último, encontramos consejos y recomendaciones dirigidos a la comunidad tendentes a la adopción de medidas higiénicas y medioambientales. Algunos de sus capítulos se dedican en su totalidad a explicar tales medidas y al papel que jugaba el enfermero en situaciones de propagación de en-

28. Opus cit. Directorio de Enfermeros, p. 216.

29. Ibídem, p. 267.

30. Ibídem, p. 260.

31. Ibídem, p. 309. 
fermedades infecciosas, como sucedía con las epidemias de peste en esos siglos. Purificar el aire, quemar la ropa contaminada, cubrir con cal los cadáveres... eran, entre otros, algunos de los consejos que debían seguirse:

«Combiene, y es muy neçesario y de mucha importançia, que en este tiempo [cuando hay epidemia de peste] no aya en las çiudades o pueblos, combentos y casas, ofiçinas de mal olor, assí como letrinas, casas de pescado, estercoleros...; también se ha de estoruar que no aya hortalizas podridas y frutas y animales muertos, porque todo esto ayuda y da más esfuerço al ayre corrupto.... $\gg^{32}$.

«No se haçe nada si, en esta ocasión de peste, no se haçen oyas hanchas y profundas en que se hechen todo género de inmundiçias... En estas oyas $y$, en acabándolas de aondar, se ha de hechar una capa de cal en poluo y, sobre ella, se han de hechar todas las inmundicias referidas y, luego al pronto, se han de cubrir de tierra y hechar otra capa de cal viba.... ${ }^{33}$.

«La segunda aduertençia o incombeniente es para los sanos, aconsexándolos que anden con grande cuydado de no beber... $\gg^{34}$.

\section{A MODO DE CONCLUSIONES}

Por su finalidad y contenido, consideramos a Directorio de Enfermeros, de Simón López, una obra clave para conocer la Enfermería español del siglo XVII. La formación del enfermero, tanto del que se iniciaba en el oficio-«prinçipiante»- como del que se encontraba ejerciendo, constituía su principal objetivo (función docente de Enfermería). A lo largo de sus páginas se aprecia un interés por delimitar el trabajo, las funciones de los profesionales que ejercían en el hospital en esa época -médico, cirujano, enfermero, barbero, boticario-, insistiéndose, sobre todo, en las propias de Enfermería.

Al explicar una determinada técnica o tarea llevada a cabo por el enfermero, el autor lo hace de forma metodológica y razonada, empleando, para su más fácil comprensión, diversos recursos didácticos (ejemplos, comparaciones, refranes). Dentro del trabajo llevado a cabo por el enfermero destaca- ba el de la promoción del autocuidado, instruyéndose para ello al enfermo en la realización de determinadas actividades y técnicas. Por último, destacamos la visión integral, biológica y sicológica, con que se contemplaba al paciente, así como la vertiente humana de los cuidados.

\section{BIBLIOGRAFÍA}

GARCÍA MARTÍNEZ, A. C.; GARCÍA MARTÍNEZ, M. J.; VALLE RACERO, J. I. (1994): «Primer Reglamento en España para la enseñanza de Practicantes y Matronas (1861)». En Qalat Chábir (Revista de Humanidades), $\mathrm{n}^{\circ} 2$, Julio de 1994. Alcalá de Guadaíra (Sevilla), pp. 123-131.

- (1997): Directorio de Enfermeros, de Simón López. Organización Colegial de Enfermería. Consejo General. Editorial Síntesis, S. A. Madrid.

GARCÍA MARTÍNEZ, A. C.; GARCÍA MARTÍNEZ, M. J.; HERNÁNDEZ MARTÍN, F.0; PÉREZ MELERO, A.; PINAR GARCÍA, M.0 E. (1993): Presentación y análisis de la obra Instrucción de Enfermeros, de Andrés Fernández, año 1625. Aproximación a la Enfermería Español de los siglos XVI-XVII. Consejo General de Colegios de Diplomados en Enfermería. Madrid.

GARCÍA MARTÍNEZ, M. J.; GARCÍA MARTÍNEZ, A. C.; VALLE RACERO, J. I. (1995): «Ficha bibliográfica ${ }^{\circ}$ 5: Directorio de Enfermeros». En Híades. Revista de Historia de la Enfermería, no 2. Alcalá de Guadaira (Sevilla), pp. 101-110.

HENDERSON, V. A. (1994): La Naturaleza de la Enfermería. Reflexiones 25 años después. InteramericanaMcGraw-Hill. Madrid.

HERNÁNDEZ MARTÍN, F.0 (Coordinadora) (1996): Historia de la Enfermería en España (desde la Antigüedad hasta nuestros días). Editorial Síntesis, S. A. Madrid.

MÍNIMA CONGREGACIÓN DE LOS HERMANOS ENFERMEROS POBRES (1625): Instrucción de Enfermeros, para aplicar los remedios a todo género de enfermedades, y acudir a muchos accidentes que sobreuienen en ausencia de los Médicos. Imprenta Real. Madrid.

ROJO VEGA, A. (1993): Enfermos y sanadores en la Castilla del siglo XVI. Servicio de Publicaciones de

32. Ibídem, p. 274.

33. Ibídem, p. 275.

34. Ibídem, p. 339. 